\title{
Neoadjuvant and adjuvant treatment approaches for hepatocellular carcinoma: future outlook
}

\author{
Ibrahim Halil Sahin ${ }^{1}$, Lana Khali1 ${ }^{2}$, Ralph Millett ${ }^{2}$, Ahmed Kaseb $^{3}$ \\ ${ }^{1}$ Moffitt Cancer Center, Tampa, FL, USA; ${ }^{2}$ Emory University School of Medicine, Winship Cancer Institute, Atlanta, GA, USA; ${ }^{3}$ Gastrointestinal \\ Medical Oncology Department, The University of Texas MD Anderson Cancer Center, Houston, TX, USA \\ Contributions: (I) Conception and design: IH Sahin, A Kaseb; (II) Administrative support: IH Sahin, A Kaseb; (III) Provision of study materials or \\ patients: IH Sahin, A Kaseb; (IV) Collection and assembly of data: All authors; (V) Data analysis and interpretation: All authors; (VI) Manuscript \\ writing: All authors; (VII) Final approval of manuscript: All authors. \\ Correspondence to: Ahmed Kaseb, MD. Professor, Gastrointestinal Medical Oncology Department, The University of Texas MD Anderson Cancer \\ Center, 1515 Holcomb Blvd, Unit 426, Houston, TX 77030, USA. Email: akaseb@mdanderson.org.
}

\begin{abstract}
Hepatocellular carcinoma (HCC) is a highly aggressive and chemotherapy resistant cancer with unique biologic characteristics which makes this disease highly different than other gastrointestinal cancers. The mainstay of curative treatment in HCC is surgical resection, ablation, and transplantation. However, rates of recurrence are high and many patients are not initially candidates for these curative approaches. This paper discusses predictors of recurrence of HCC in patients who have undergone surgical resection and addresses adjuvant therapies aimed at decreasing recurrence risk and improving overall survival (OS) outcomes, including traditional cytotoxic chemotherapies, tyrosine kinase inhibitors (TKIs), and immunotherapy. This article also discusses neoadjuvant strategies aimed at improving recurrence rate and OS as well as downstaging advanced HCC to enable surgical disease, including locoregional therapies, systemic chemotherapy, TKIs, and immune checkpoint inhibitors. Finally, this article addresses potential future directions for both adjuvant and neoadjuvant therapies that may change the treatment paradigm of $\mathrm{HCC}$ in the near future.
\end{abstract}

Keywords: Hepatocellular carcinoma (HCC); neoadjuvant treatment; adjuvant treatment

Submitted Dec 12, 2020. Accepted for publication Dec 22, 2020.

doi: $10.21037 / \mathrm{cco}-20-248$

View this article at: http://dx.doi.org/10.21037/cco-20-248

\section{Introduction}

Hepatocellular carcinoma (HCC) is the second leading cause of cancer related mortality worldwide with an estimated global incidence of between 500,000-1,000,000 and an attributable 600,000 deaths annually (1). Unfortunately, HCC is also increasing faster than any other cancer for both men and women in the United States despite the fact that roughly $70 \%$ of cases develop secondary to modifiable risk factors (e.g., alcohol use, chronic viral hepatitis infections, obesity, and smoking) (2). Of unique challenge in the treatment HCC is the high co-occurrence of underlying liver disease which inherently limits potential systemic therapeutic approaches, and despite progress in the management and treatment options of HCC in the last decades, the prognosis remains dismal with a 5 -year survival of less than $5 \%$ (3).

While treatment of HCC is multidisciplinary, at present the only therapies directed at cure are aimed at physical removal or destruction of local disease. In patients with relatively preserved liver function, surgical resection is the mainstay treatment but is often limited by the size and anatomy of the disease $(4,5)$. In patients with end-stage liver disease (ESLD), liver transplantation (LT) is considered the optimal treatment option as it addresses both the malignancy and the underlying cirrhosis (6). Transplanted patients with localized HCC have a have 5-year survival rates of between $65-80 \%$ with a tumor recurrence rate 
between of $8-15 \%$ (7). Unfortunately, most patients with HCC are ineligible for transplantation by Milan Criteria at the time of presentation and organ procurement remains a challenge even for those who are initially transplant candidates (6-8). As a result, many of the treatment options discussed in this paper are directed at downstaging or stabilization of disease in advance of potential resection or transplantation, in many cases by applying therapies otherwise approved for the systemic treatment of unresectable or metastatic HCC.

In this review article, we discuss predictors of recurrence among surgically resected patients with HCC and present an overview of the recent progress in different therapeutic approaches aimed at reducing the risk of recurrence and improving overall survival (OS) outcomes. We also evaluate the efficacy of neoadjuvant and adjuvant approaches with novel approaches including immunotherapy as well as targeted therapies and provide a perspective on the likely future direction of clinical trials and treatment options in this highly challenging cancer.

\section{Predictors of recurrence in HCC patients}

Given the aggressive nature of HCC, many studies have explored potential clinical and molecular predictors of recurrence. In a study of 112 patients who underwent curative partial hepatectomy, tumor size, number, capsular invasion, and portal vein involvement were identified as a predictor of recurrence (9). A separate study in an Asian cohort corroborated these same features as predictors of recurrence after curative surgery and suggested the use of baseline serum $\alpha$-fetoprotein and albumin levels as surrogates of recurrence (10). Another clinical study in patients who underwent curative resection suggested that microvascular invasion may be a better surrogate of risk of recurrence as compared to the Milan criteria. Similarly, tumor microvessel density measured by immunohistochemical analysis of CD34 has been described as a predictor of recurrence among patients with HCC, though this correlation was found only in tumors $<5 \mathrm{~cm}$ $(11,12)$. CD24, a cancer stem cell marker found to be overexpressed in various tumors, has been investigated in patients with HCC with a reportedly increased metastatic potential with activation of the $\mathrm{Wnt} / \beta$-catenin pathway leading to higher rates of intrahepatic and distant recurrence in surgically resected patients (13). In the same study, CD24 expression was associated with a poor OS outcomes outcome independent of $\alpha$-fetoprotein, a tumor- node-metastasis stage.

Inflammatory markers have also been investigated as surrogates of recurrence in HCC patients. CD68+ tumorinfiltrating macrophages have been noted in the tumor microenvironment of HCC and in a study of 137 surgically resected patients with HCC, higher intratumoral and tumor margin macrophage infiltration was associated with a shorter disease-free survival (14). Increased circulating neutrophils relative to lymphocytes may also be associated with an increased risk of recurrence (15-17), however it is important to note that the neutrophil: lymphocyte ratio is a subjective and dynamic marker affected by multiple confounding factors which might otherwise co-occur in this patient population (e.g., steroid use, infection). It is posited that elevation in these inflammatory markers may reflect underlying systemic inflammatory responses which in turn have been shown to fuel angiogenesis and cancer growth (18).

In a study of 302 patients with HCC who underwent hepatectomy, high intratumoral levels of cytotoxic $\mathrm{T}$ cells and low levels of $\mathrm{T}$ regulatory cell were associated with a better 5 -year disease-free survival along with improved OS (19). Another study found that elevated levels of programmed cell death ligand-1 (PD-L1) were associated with an increased risk of recurrence when overexpressed in HCC (20). In another translational study, improved time to recurrence was noted with an increased CD4 T cell signature in the tumor microenvironment among 42 patients with HCC (21). Interestingly, although memory CD8+ $\mathrm{T}$ cells were associated with a lower risk of recurrence, effector CD8+ $\mathrm{T}$ cell signature had a higher risk of recurrence (21). The authors also identified a shorter time to relapse with increased expression of PD-L1 in the tumor tissue (21). Collectively, this evidence suggests that T-cell phenotype, as well as PD-L1 expression, may be an important factor for the recurrence of HCC after curative resection.

\section{Adjuvant approaches}

\section{Cytotoxic agents}

Surgical resection is one of the primary curative-intent options in HCC with many neoadjuvant treatments aimed at downstaging tumors in advance of resection. Unfortunately, there is an approximately $70 \%$ risk of recurrence at 5 years after resection (22), and for this reason adjuvant chemotherapy (AC) has been explored as a 
method to improve recurrence free survival (RFS) and OS both after partial hepatectomy as well as liver transplant. Some of these AC approaches have evaluated concurrent systemic and locally directed chemotherapeutic agents. In an early randomized clinical trial, investigators evaluated the combination of systemic epirubicin hydrochloride with transarterial chemotherapy using an emulsion of cisplatin and iodized oil (23). The authors observed detrimental outcomes among patients who underwent AC compared to the control arm and noted a higher incidence of distant recurrence. Another trial evaluated the combination of intrahepatic epirubicin infusion with systemic uraciltegafur in patients' post-radical hepatic resection (24). This intervention showed no improvement in the clinical outcome nor in risk of recurrence (24). Similarly, a metaanalysis evaluating the use of systemic and intrahepatic arterial epirubicin infusion in combination with systemic carmofur showed no statistically significant difference in disease-free survival outcomes at 2 years (25).

Adjuvant intrahepatic approaches without concurrent systemic therapies have also been explored. In a prospective trial of 50 patients with HCC, hepatic arterial infusion of lipiodol containing doxorubicin and mitomycin $\mathrm{C}$ was investigated after hepatectomy (26). Although this approach moderately reduced the risk of recurrence, it did not improve OS outcomes. Adjuvant 5-fluorouracil in combination with cisplatin infused through the hepatic artery similarly did not demonstrate beneficial effect (27).

AC has also been studied following LT for HCC. A study exploring use of post-transplant cisplatin and gemcitabine suggested improvement in disease-free survival in patients who did not Milan criteria after transplant (28). A similar study conducted with FOLFOX (5-fluorouracil, leucovorin, and oxaliplatin) in patients who underwent upfront LT also showed benefit in patients who did not meet Milan Criteria after transplant (29). Yet another clinical trial of triple agent chemotherapy with 5 -fluorouracil, cisplatin, and doxorubicin showed a significantly improved diseasefree survival at 3-year remark, though notably the control arm utilized historical data which limits extrapolation to real populations (30). On the obverse, a randomized study investigating the efficacy of perioperative doxorubicin in 62 post-transplant HCC patients failed to demonstrate beneficial outcome (31). In fact, disease-free survival rates for the intervention arm $v$ s. control arm were $43 \%$ and $53 \%$ at 5 years, suggesting numerically worse outcomes with the use of chemotherapy (31). Consistent with that trial, multiple meta-analyses have also failed to show significant improvement in outcomes with the use of AC in HCC patients (32-34). Collectively, the current data do not support the use of AC in patients with HCC after curative surgical resection or transplantation.

\section{Tyrosine kinase inbibitors (TKIs)}

TKIs have achieved substantial success in many solid tumor types including HCC $(8,9,35,36)$. Sorafenib, an oral multikinase inhibitor which inhibits platelet-derived growth factor (PDGF) and vascular endothelial growth factors (VEGF) 1-3 was the first TKI approved for the treatment of advanced-stage HCC (36). It has since been investigated as adjuvant treatment with early small studies demonstrating promising results (37). In a study of 31 HCC patients who underwent upfront surgical resection, 14 patients who received adjuvant sorafenib demonstrated a significantly lower incidence of recurrence compared against the control group (29.4\% vs. $70.7 \%, \mathrm{P}=0.032)$ (38). This benefit was not reproducible in larger cohorts, however. The STORM trial, a randomized double-blind placebo-controlled phase III trial, measured RFS with adjuvant sorafenib after resection or ablation of HCC. No significant difference was found in median RFS between sorafenib vs. placebo (33.3 vs. 33.7 months, respectively) and there was an increased rate of adverse effects in the sorafenib arm (39). Ongoing studies include investigation of sorafenib in combination with systemic chemotherapy (NCT02129322) and with chemoembolization approaches (NCT04143191) as well as use of adjuvant apatinib after hepatectomy for prevention of tumor recurrence (NCT03261791). At this time, TKIs are not standard of care adjuvant treatment for HCC.

\section{Antiviral treatment}

Hepatitis C virus (HCV) and hepatitis B virus (HBV) play an outsized role in the development of cirrhosis and are found in $80-90 \%$ of patients with HCC (6). Evidence indicates that uncontrolled $\mathrm{HBV} / \mathrm{HCV}$ infections lead to increased rates of hepatocarcinogenesis and decreased liver remnant function after resection of HCC. In patients with $\mathrm{HBV}$-related HCC, for instance, a prospective cohort study examined patients with high viral loads $(>10,000$ copies $/ \mathrm{mL}$ HBV DNA) vs. low viral loads $(<10,000$ copies $/ \mathrm{mL} \mathrm{HBV}$ DNA) after resection of HCC and demonstrated that subjects with a higher viral loads had lower median OS (78.3 vs. 111.4 months, $\mathrm{P}<0.001)$ as well as RFS (44.6 vs. 94.8 months, $\mathrm{P}<0.001)(40)$. 
To that end, adjuvant antiviral treatments using interferon-based regimens have been studied extensively with the aim of increasing RFS after partial hepatectomy. Smaller studies have demonstrated mixed RFS benefits with adjuvant interferon therapy but were notably heterogeneous in the antiviral regimen used (41-43). A meta-analysis of nine randomized trials and four cohort studies $(1,180$ subjects) suggested improvement in 1-, 2-, and 3-year RFS (7.8\%, $35.4 \%$, and $14 \%$ respectively) in $\mathrm{HBV}$ and $\mathrm{HCV}$ infected patients who received adjuvant interferon therapy after curative therapy (44). Another meta-analysis of four cohort studies (1,280 patients) demonstrated improved 3and 5-year RFS as well as 5-year OS with use of pegylatedinterferon treatment as adjuvant treatment (45). However, a systematic review of six trials ( 1,054 subjects) failed to demonstrate a statistically significant difference in recurrence of HCC after surgical resection (43). At this time, adjuvant interferon treatment is not standard of care.

Studies have also examined the role of newer nucleotide/ nucleoside inhibitors (NAs) as adjuvant treatment HCC. A two-stage longitudinal study evaluating 780 patients with HBV-related HCC (163 in RCT, 617 in nonRCT) looked at post-operative outcomes in patients who received adjuvant lamivudine, adefovir dipivoxil, or entecavir (all nucleotide/nucleoside analogs). Patients who received antiviral treatment had significantly decreased early recurrence 6 months as well as a higher 2 -year RFS (46). Meanwhile, a meta-analysis of 13 trials (6,350 patients) who received adjuvant NAs in HBV-related HCC demonstrated significant improvements in both RFS and OS (47). Interestingly, while a retrospective cohort study of patients with HCV-related HCC has also demonstrated a significant reduction in risk of death in patients with sustained virologic response in the adjuvant setting, there is less overall evidence for adjuvant NA use in cancers related to HCV (48).

\section{Immunotherapeutic approaches}

Immunotherapy has been explored as a method by which to reduce the risk of recurrence in patients with resected HCC (49). A randomized trial of 150 post-resection HCC patients investigated use of adjuvant adoptive immunotherapy and demonstrated a significantly longer time to recurrence in patients treated with immunotherapy compared to the control group ( $48 \%$ vs. $33 \%$ at 3 years, $38 \%$ vs. $22 \%$ at 5 years; $\mathrm{P}=0.008$ ) (50). In an open-label, phase 3 trial of activated autologous cytokine-induced killer cells, (CD3+/CD56+ and CD3+/CD56- T cells and CD3-/CD56+ natural killer cells), there was a reported improvement in RFS by 14 months as compared to the control group as well as improved OS (HR, 0.19; 95\% CI, 0.04-0.87; $\mathrm{P}=0.02$ ) (51). This same benefit was suggested in a retrospective review of patients treated with cytokineinduced killer cells (52). Notably, the authors reported PD-1 expressing tumor-infiltrating lymphocytes as a biomarker of response. In an early phase study of tumor antigen-pulsed dendritic cells, 12 patients with HCC who underwent liver resection were injected with dendritic cell vaccines pulsed with different cancer antigens including cytoplasmic transduction peptide-attached alpha-fetoprotein glypican-3 and melanoma-associated antigen 1 recombinant fusion (53). This approach was found to be safe and showed some early signals. Even though cellular therapy with cancer vaccines appears to promise moderate signal for reduction of recurrence risk, further safety studies and randomized trials are needed to better understand the role of these agents in patients with HCC (54).

More recently clinical trials have investigated the use of adjuvant immune checkpoint inhibitors in patients with HCC undergoing liver resections (55). Pembrolizumab, a fully humanized monoclonal antibody against PD-1, is being investigated in a double-blind randomized phase III trial in the adjuvant setting (NCT03867084). Another current study is investigating RFS in patients with HCC who have achieved complete response after local ablation or complete resection, and who are high-risk, on nivolumab compared to placebo. A phase II trial study (NCT03222076) has enrolled 30 patients with patients resectable HCC in the setting of presurgical and pre-biopsy therapy to evaluate the safety and tolerability of therapy with nivolumab alone or with ipilimumab. Also, neoadjuvant cabozantinib plus nivolumab will be evaluated in patients with locally advanced HCC in an open-label, single institution, single arm phase 1b study (NCT03299946). Adjuvant atezolizumab and bevacizumab is also being investigated in a phase III trial of high-risk HCC patients who underwent a curative treatment (NCT04102098). Growing interest also exists for the use of these agents in the perioperative setting which is further discussed in the neoadjuvant section below.

\section{Neoadjuvant approaches}

\section{Liver-directed therapies}

Malignant transformation of liver tissue augments arterial 
blood flow relative to healthy parenchyma, thereby increasing susceptibility of HCC to arterially directed therapies relative to surrounding healthy liver tissue (56). Transarterial chemoembolization (TACE) is a commonly used approach that takes advantage of this phenomenon by injecting small amounts of chemotherapeutic agents into arteries directly supplying tumors and can be used to downstage locally advanced HCC prior to surgical resection. A retrospective analysis of $831 \mathrm{HCC}$ patients who underwent TACE for unresectable HCC reported that $82(\sim 10 \%)$ of these patients were downstaged to resectable disease, $43(\sim 5 \%)$ of whom underwent salvage liver resection (57). In patients who received a PR with TACE, patients who underwent salvage liver resection had an OS of 49 months compared to 24 months in those who did not undergo surgery $(\mathrm{P}<0.001)$; there did not appear to be survival advantage to liver resection if a $\mathrm{CR}$ was obtained with TACE (50 vs. 54 months).

A separate retrospective analysis of 1,457 patients revealed improved 5 -year disease-free survival in patients who underwent neoadjuvant TACE $(51 \%, 35.5 \%$, and $21.4 \%$ for multiple TACE procedures, one TACE procedure, and no TACE, respectively) $(58,59)$. Notably, a systematic review which included eighteen studies (15 observational, three randomized) demonstrated high rates of pathological responses but did not demonstrate improvement in DFS and was unable to reach a conclusion with respect to OS (60). Nonetheless, unresectable HCC patients who are downstaged with TACE and followed by salvage surgical resection overall appear to have better outcomes, suggesting that TACE is a viable option in borderline resectable HCC patients who are otherwise good surgical candidates with reasonable future liver remnant (61).

TACE is also utilized to downstage patients who do not initially meet Milan criteria but are otherwise viable transplant candidates. In a prospective study of 76 patients who received pre-transplant TACE, 17 were successfully down staged and transplanted and at the time of median follow up (19.6 months), there was only a single tumor recurrence (61). TACE may also be used to achieve tumor control in those undergoing evaluation for and awaiting transplantation (62).

Transarterial radioembolization (TARE) is an alternative locoregional treatment increasingly being implemented in the neoadjuvant setting. Similar to TACE, TARE is arterially directed but instead uses Y-90 resin microspheres for local irradiation of tumor cells (selective internal radiation therapy-SIRT) (63). Uniquely, TARE can be utilized in patients with portal vein thromboses $(64,65)$ and also induces contralateral liver lobe (future liver remnant) hypertrophy in unilobar tumors which may potentiate liver recovery after salvage liver resection $(66,67)$. Evidence suggests that TARE may even lead to better local disease control than to TACE. A retrospective analysis of 463 HCC patients comparing clinical outcomes of TARE and TACE demonstrated superior progression-free survival (13.3 vs. 8.4 months, $\mathrm{P}=0.046$ ) with the use of TARE, though notably though median OS was similar (20.5 vs. 17.4 months, $\mathrm{P}=0.232$ ) (63). A separate comparative analysis of TARE $v s$. TACE for downstaging HCC prior to LT reported a significantly higher objective response in patients in the TARE arm (61\% vs. $37 \%$ respectively). Event-free survival was likewise significantly improved (17.7 months for TARE vs. 7.1 months for TACE, $\mathrm{P}=0.0017)$ as was uncensored OS (41.6 for TARE $v$. 19.2 months for TACE; $\mathrm{P}=0.008$ ) (68). Overall, this evidence demonstrates the growing role of TARE in downstaging HCC prior to salvage surgery or orthotopic liver transplant.

\section{Cytotoxic agents}

Systemic treatment options for unresectable HCC have been historically few and largely ineffective. In particular, cytotoxic chemotherapy has historically played little role as either definitive or neoadjuvant treatment for unresectable HCC. This is in large part attributed to the dose-limiting toxicities of cytotoxic regimens in patients with underlying liver disease and the subsequently decreased rates of downstaging as compared to liver-directed therapies (69). A retrospective study evaluating GEMOX (gemcitabine, oxaliplatin) demonstrated that $8.5 \%$ of patients became eligible for curative-intent treatment strategies and had favorable toxicity compared to doxorubicin monotherapy (70). In a phase II trial, another regimen known as PIAF (cisplatin, interferon-alpha-2b, doxorubicin, and 5 -fluorouracil) demonstrated an $18 \%$ conversion rate to curative surgery (71). The phase III investigation, however, downstaged only $8 \%$ of participants in the PIAF arm to surgical resectability and reported considerable and doselimiting toxicity with the regimen (72).

Notably, however, selective application of chemotherapy may yet play a role in the neoadjuvant setting. A prospective cohort study examined a modified PIAF regimen in patients who did not have underlying liver disease beyond HCC. 
Promisingly, it demonstrated a conversation rate to curative surgery of $33 \%$ (as well as an objective response rate of $36 \%$ and median OS of 21.3 months) (73), indicating future areas of study in patients without underlying hepatic dysfunction.

\section{TKIs}

In addition to use in unresectable and metastatic HCC, sorafenib has also been explored as neoadjuvant therapy prior to liver transplant with multiple case reports and case series suggesting it as a cost-effective bridging therapy during the time between diagnosis and transplantation (74-76). At least one prospective trial has failed to find any benefit to sorafenib monotherapy prior to transplantation (77), however, and it is not currently approved in the neoadjuvant setting. Interestingly, a metaanalysis of 17 publications suggests that sorafenib combined with TACE may improve time to progression in the pretransplant setting indicating area for further study (78).

\section{Immune checkpoint inbibitors}

Malignant cells may evade detection by the immune system by upregulation of cell-surface immune checkpoints. Checkpoint inhibitors function by unmasking these cells and potentiating immune responses against them and have been utilized in a growing number of solid cancers including HCC. A combination of atezolizumab (PD-L1 inhibitor) and bevacizumab (VEGF A inhibitor) is currently approved for first-line treatment of unresectable or metastatic HCC (79), and checkpoint inhibitors are now under investigation as neoadjuvant treatment for HCC.

A phase II randomized trial of nivolumab (a fullyhumanized monoclonal anti-PD-1 antibody) and nivolumab plus ipilimumab (a monoclonal anti-CTLA4 antibody) as preoperative treatment in resectable HCC patients has reported pathologic complete response in $25 \%$ of patients studied (5 patients out of 20) without delay of surgery (55). Another recent trial is an open-label single arm phase $1 \mathrm{~b}$ study investigating neoadjuvant cabozantinib plus nivolumab in patients with locally advanced HCC. Pembrolizumab, another PD-1 inhibitor, is likewise currently under investigation as perioperative therapy prior to curative treatment (hepatic resection or radiofrequency ablation) (80). Pembrolizumab may also have a role in downstaging in the pre-transplant setting given a $16.2 \%$ partial response rate in the phase 3 KEYNOTE-240 trial (80), though the use of immunotherapy immediately prior to transplant is problematic due to increased risk of organ rejection.

Combination therapies involving immune checkpoint inhibitors and small molecule in the neoadjuvant setting are also under investigation, including the use of nivolumab and cabozantinib prior to curative therapy (NCT03299946) as well as pembrolizumab and lenvatinib prior to liver transplant (NCT0445226).

\section{Discussion and future perspective}

The current state of science discussed above has yet to demonstrate sufficient evidence to recommend any agent as neoadjuvant or adjuvant therapy in patients with HCC who are candidates for surgical resection or curative therapy with locoregional approaches. However, as noted above, locally advanced or borderline resectable patients should be considered for liver-directed therapy, perhaps particularly with TARE over TACE based on the advantages discussed above; by downstaging the primary tumor to surgically resectable disease. These patients should have considerably reasonable future liver remnant with liver function (preferably Child-Pugh Class A). Systemic chemotherapy and TKIs have been investigated extensively in patients with HCC after curative treatments. At this time, cytotoxic agents don't appear to be beneficial, and are perhaps detrimental in a subgroup of HCC patients. TKIs, also, have a limited role in the adjuvant and neoadjuvant setting, as the role of single agents in combination with immune checkpoint inhibitors deserves attention.

Although there is no systemic therapy option approved for neoadjuvant or adjuvant therapy, the future is promising for immune checkpoint inhibitors within different modalities. The early findings of nivolumab and nivolumab plus ipilimumab in the perioperative setting will likely change the current paradigm in the near future. The use of immune checkpoint therapy, in combination with locoregional treatments, which hypothetically increase neoantigen release from ablated tumors, may enhance the potential benefits of these agents. IMbrave 150 trial (7) showed a synergistic effect of angiogenesis inhibitors with immune checkpoint inhibitors and further studies with this approach may lead to practice-changing results. Although these promising results keep these agents in the exciting future concepts, further studies are warranted to better understand the exact clinical effect of observed promising objective response on survival outcomes including diseasefree survival and OS. 
Summing up, the studies discussed above hold promises for the future of neoadjuvant and adjuvant therapeutic options and perhaps with immunotherapy-based therapeutic choices. It is important to note that, we do not have clinical and molecular markers for immune response in patients with HCC, and therefore future studies should also interrogate surrogates of response to immune checkpoint inhibitors in patients with HCC to better identify target patient populations who may benefit from these novel approaches. Prospective clinical trials should also strongly consider including correlative studies to better characterize the anti-cancer immune response which may further change our current understanding and will subsequently change the current practice of resectable tumor in patients with HCC in the near future.

\section{Acknowledgments}

Funding: None.

\section{Footnote}

Provenance and Peer Review: This article was commissioned by the Guest Editors (Mehmet Akce and Shishir K. Maithel) for the series "Hepatocellular Carcinoma" published in Chinese Clinical Oncology. The article was sent for external peer review organized by the Guest Editors and the editorial office.

Conflicts of Interest: All authors have completed the ICMJE uniform disclosure form (available at http://dx.doi. org/10.21037/cco-20-248). The series "Hepatocellular Carcinoma" was commissioned by the editorial office without any funding or sponsorship. The authors have no other conflicts of interest to declare.

Ethical Statement: The authors are accountable for all aspects of the work in ensuring that questions related to the accuracy or integrity of any part of the work are appropriately investigated and resolved.

Open Access Statement: This is an Open Access article distributed in accordance with the Creative Commons Attribution-NonCommercial-NoDerivs 4.0 International License (CC BY-NC-ND 4.0), which permits the noncommercial replication and distribution of the article with the strict proviso that no changes or edits are made and the original work is properly cited (including links to both the formal publication through the relevant DOI and the license). See: https://creativecommons.org/licenses/by-nc-nd/4.0/.

\section{References}

1. Teh SH, Christein J, Donohue J, et al. Hepatic resection of hepatocellular carcinoma in patients with cirrhosis: Model of End-Stage Liver Disease (MELD) score predicts perioperative mortality. J Gastrointest Surg 2005;9:120715; discussion 1215.

2. Siegel RL, Miller KD, Jemal A. Cancer statistics, 2019. CA Cancer J Clin 2019;69:7-34.

3. Desai A, Sandhu S, Lai JP, et al. Hepatocellular carcinoma in non-cirrhotic liver: a comprehensive review. World J Hepatol 2019;11:1-18.

4. Livraghi T, Meloni F, Di Stasi M, et al. Sustained complete response and complications rates after radiofrequency ablation of very early hepatocellular carcinoma in cirrhosis: Is resection still the treatment of choice? Hepatology 2008;47:82-9.

5. Kow AWC. Transplantation versus liver resection in patients with hepatocellular carcinoma. Transl Gastroenterol Hepatol 2019;4:33.

6. Saidi RF, Hejazi Kenari SK. Liver transplantation for hepatocellular carcinoma: past, present and future. Middle East J Dig Dis 2013;5:181-92.

7. Duvoux C, Kiuchi T, Pestalozzi B, et al. What is the role of adjuvant therapy after liver transplantation for hepatocellular carcinoma? Liver Transpl 2011;17 Suppl 2:S147-58.

8. Rubin J, Ayoub N, Kaldas F, et al. Management of recurrent hepatocellular carcinoma in liver transplant recipients: a systematic review. Exp Clin Transplant 2012;10:531-43.

9. Arii S, Tanaka J, Yamazoe Y, et al. Predictive factors for intrahepatic recurrence of hepatocellular carcinoma after partial hepatectomy. Cancer 1992;69:913-9.

10. Hao K, Luk JM, Lee NP, et al. Predicting prognosis in hepatocellular carcinoma after curative surgery with common clinicopathologic parameters. BMC Cancer 2009;9:389.

11. Poon RT, Ng IO, Lau C, et al. Tumor microvessel density as a predictor of recurrence after resection of hepatocellular carcinoma: a prospective study. J Clin Oncol 2002;20:1775-85.

12. Lim KC, Chow PK, Allen JC, et al. Microvascular invasion is a better predictor of tumor recurrence and overall survival following surgical resection for hepatocellular 
carcinoma compared to the Milan criteria. Ann Surg 2011;254:108-13.

13. Yang XR, Xu Y, Yu B, et al. CD24 is a novel predictor for poor prognosis of hepatocellular carcinoma after surgery. Clin Cancer Res 2009;15:5518-27.

14. Ding T, Xu J, Wang F, et al. High tumor-infiltrating macrophage density predicts poor prognosis in patients with primary hepatocellular carcinoma after resection. Hum Pathol 2009;40:381-9.

15. Yamamura K, Sugimoto H, Kanda M, et al. Comparison of inflammation-based prognostic scores as predictors of tumor recurrence in patients with hepatocellular carcinoma after curative resection. J Hepatobiliary Pancreat Sci 2014;21:682-8.

16. Mano Y, Shirabe K, Yamashita Y, et al. Preoperative neutrophil-to-lymphocyte ratio is a predictor of survival after hepatectomy for hepatocellular carcinoma: a retrospective analysis. Ann Surg 2013;258:301-5.

17. Hu B, Yang XR, Xu Y, et al. Systemic immuneinflammation index predicts prognosis of patients after curative resection for hepatocellular carcinoma. Clin Cancer Res 2014;20:6212-22.

18. Coussens LM, Werb Z. Inflammation and cancer. Nature 2002;420:860-7.

19. Gao Q, Qiu SJ, Fan J, et al. Intratumoral balance of regulatory and cytotoxic $\mathrm{T}$ cells is associated with prognosis of hepatocellular carcinoma after resection. J Clin Oncol 2007;25:2586-93.

20. Gao Q, Wang XY, Qiu SJ, et al. Overexpression of PDL1 significantly associates with tumor aggressiveness and postoperative recurrence in human hepatocellular carcinoma. Clin Cancer Res 2009;15:971-9.

21. Cariani E, Pilli M, Zerbini A, et al. Immunological and molecular correlates of disease recurrence after liver resection for hepatocellular carcinoma. PLoS One 2012;7:e32493.

22. Allaire M, Goumard C, Lim C, et al. New frontiers in liver resection for hepatocellular carcinoma. JHEP Rep 2020;2:100134.

23. Lai EC, Lo CM, Fan ST, et al. Postoperative adjuvant chemotherapy after curative resection of hepatocellular carcinoma: a randomized controlled trial. Arch Surg 1998;133:183-8.

24. Kohno H, Nagasue N, Hayashi T, et al. Postoperative adjuvant chemotherapy after radical hepatic resection for hepatocellular carcinoma (HCC). Hepatogastroenterology 1996;43:1405-9.

25. Ono T, Nagasue N, Kohno H, et al. Adjuvant chemotherapy with epirubicin and carmofur after radical resection of hepatocellular carcinoma: a prospective randomized study. Semin Oncol 1997;24:S6-18-S6-25.

26. Izumi R, Shimizu K, Iyobe T, et al. Postoperative adjuvant hepatic arterial infusion of Lipiodol containing anticancer drugs in patients with hepatocellular carcinoma. Hepatology 1994;20:295-301.

27. Kim DY, Ahn SH, Kim SU, et al. Adjuvant hepatic arterial infusional chemotherapy with 5-fluorouracil and cisplatin after curative resection of hepatocellular carcinoma. Oncology 2011;81:184-91.

28. Hsieh CB, Chou SJ, Shih ML, et al. Preliminary experience with gemcitabine and cisplatin adjuvant chemotherapy after liver transplantation for hepatocellular carcinoma. Eur J Surg Oncol 2008;34:906-10.

29. Zhang Q, Chen H, Li Q, et al. Combination adjuvant chemotherapy with oxaliplatin, 5-fluorouracil and leucovorin after liver transplantation for hepatocellular carcinoma: a preliminary open-label study. Invest New Drugs 2011;29:1360-9.

30. Olthoff KM, Rosove MH, Shackleton CR, et al. Adjuvant chemotherapy improves survival after liver transplantation for hepatocellular carcinoma. Ann Surg 1995;221:734-41; discussion 731-43.

31. Pokorny H, Gnant M, Rasoul-Rockenschaub S, et al. Does additional doxorubicin chemotherapy improve outcome in patients with hepatocellular carcinoma treated by liver transplantation? Am J Transplant 2005;5:788-94.

32. Samuel M, Chow PK, Chan Shih-Yen E, et al. Neoadjuvant and adjuvant therapy for surgical resection of hepatocellular carcinoma. Cochrane Database Syst Rev 2009;2009:CD001199.

33. Zhu GQ, Shi KQ, Yu HJ, et al. Optimal adjuvant therapy for resected hepatocellular carcinoma: a systematic review with network meta-analysis. Oncotarget 2015;6:18151-61.

34. Ono T, Yamanoi A, Nazmy El Assal O, et al. Adjuvant chemotherapy after resection of hepatocellular carcinoma causes deterioration of long-term prognosis in cirrhotic patients: metaanalysis of three randomized controlled trials. Cancer 2001;91:2378-85.

35. Guler I, Askan G, Klostergaard J, et al. Precision medicine for metastatic colorectal cancer: an evolving era. Expert Rev Gastroenterol Hepatol 2019;13:919-31.

36. Llovet JM, Ricci S, Mazzaferro V, et al. Sorafenib in advanced hepatocellular carcinoma. N Engl J Med 2008;359:378-90.

37. Shetty K, Dash C, Laurin J. Use of adjuvant sorafenib in liver transplant recipients with high-risk hepatocellular 
carcinoma. J Transplant 2014;2014:913634.

38. Wang SN, Chuang SC, Lee KT. Efficacy of sorafenib as adjuvant therapy to prevent early recurrence of hepatocellular carcinoma after curative surgery: a pilot study. Hepatol Res 2014;44:523-31.

39. Bruix J, Takayama T, Mazzaferro V, et al. Adjuvant sorafenib for hepatocellular carcinoma after resection or ablation (STORM): a phase 3, randomised, double-blind, placebo-controlled trial. Lancet Oncol 2015;16:1344-54.

40. Lo CM, Liu CL, Chan SC, et al. A randomized, controlled trial of postoperative adjuvant interferon therapy after resection of hepatocellular carcinoma. Ann Surg 2007;245:831-42.

41. Ikeda K, Arase Y, Saitoh S, et al. Interferon beta prevents recurrence of hepatocellular carcinoma after complete resection or ablation of the primary tumor-A prospective randomized study of hepatitis $\mathrm{C}$ virus-related liver cancer. Hepatology 2000;32:228-32.

42. Lin SM, Lin CJ, Hsu CW, et al. Prospective randomized controlled study of interferon-alpha in preventing hepatocellular carcinoma recurrence after medical ablation therapy for primary tumors. Cancer 2004;100:376-82.

43. Chen LT, Chen MF, Li LA, et al. Long-term results of a randomized, observation-controlled, phase III trial of adjuvant interferon Alfa-2b in hepatocellular carcinoma after curative resection. Ann Surg 2012;255:8-17.

44. Shen YC, Hsu C, Chen LT, et al. Adjuvant interferon therapy after curative therapy for hepatocellular carcinoma (HCC): a meta-regression approach. J Hepatol 2010;52:889-94.

45. Wu J, Yin Z, Cao L, et al. Adjuvant pegylated interferon therapy improves the survival outcomes in patients with hepatitis-related hepatocellular carcinoma after curative treatment: a meta-analysis. Medicine (Baltimore) 2018;97:e11295.

46. Yin J, Li N, Han Y, et al. Effect of antiviral treatment with nucleotide/nucleoside analogs on postoperative prognosis of hepatitis B virus-related hepatocellular carcinoma: a two-stage longitudinal clinical study. J Clin Oncol 2013;31:3647-55.

47. Sun P, Dong X, Cheng X, et al. Nucleot(s)ide analogues for hepatitis B virus-related hepatocellular carcinoma after curative treatment: a systematic review and meta-analysis. PLoS One 2014;9:e102761.

48. Singal AG, Rich NE, Mehta N, et al. Direct-acting antiviral therapy for hepatitis $\mathrm{C}$ virus infection is associated with increased survival in patients with a history of hepatocellular carcinoma. Gastroenterology 2019;157:1253-63.e2.

49. Hui D, Qiang L, Jian W, et al. A randomized, controlled trial of postoperative adjuvant cytokine-induced killer cells immunotherapy after radical resection of hepatocellular carcinoma. Dig Liver Dis 2009;41:36-41.

50. Takayama T, Sekine T, Makuuchi M, et al. Adoptive immunotherapy to lower postsurgical recurrence rates of hepatocellular carcinoma: a randomised trial. Lancet 2000;356:802-7.

51. Lee JH, Lee JH, Lim YS, et al. Adjuvant immunotherapy with autologous cytokine-induced killer cells for hepatocellular carcinoma. Gastroenterology 2015;148:1383-91.e6.

52. Chang B, Shen L, Wang K, et al. High number of PD-1 positive intratumoural lymphocytes predicts survival benefit of cytokine-induced killer cells for hepatocellular carcinoma patients. Liver Int 2018;38:1449-58.

53. Lee JH, Lee Y, Lee M, et al. A phase I/IIa study of adjuvant immunotherapy with tumour antigen-pulsed dendritic cells in patients with hepatocellular carcinoma. Br J Cancer 2015;113:1666-76.

54. Xie F, Zhang X, Li H, et al. Adoptive immunotherapy in postoperative hepatocellular carcinoma: a systemic review. PLoS One 2012;7:e42879.

55. Kaseb AO, Carmagnani Pestana R, Vence LM, et al. Randomized, open-label, perioperative phase II study evaluating nivolumab alone versus nivolumab plus ipilimumab in patients with resectable HCC. J Clin Oncol 2019;37:185.

56. Matsui O, Kadoya M, Kameyama T, et al. Benign and malignant nodules in cirrhotic livers: distinction based on blood supply. Radiology 1991;178:493-7.

57. Zhang Y, Huang G, Wang Y, et al. Is salvage liver resection necessary for initially unresectable hepatocellular carcinoma patients downstaged by transarterial chemoembolization? Ten years of experience. Oncologist 2016;21:1442-9.

58. Zhang Z, Liu Q, He J, et al. The effect of preoperative transcatheter hepatic arterial chemoembolization on disease-free survival after hepatectomy for hepatocellular carcinoma. Cancer 2000;89:2606-12.

59. Qi X, Wang D, Su C, et al. Hepatic resection versus transarterial chemoembolization for the initial treatment of hepatocellular carcinoma: a systematic review and metaanalysis. Oncotarget 2015;6:18715-33.

60. Chapman WC, Majella Doyle MB, et al. Outcomes of neoadjuvant transarterial chemoembolization to downstage hepatocellular carcinoma before liver transplantation. Ann 
Surg 2008;248:617-25.

61. Lau WY, Lai EC. Salvage surgery following downstaging of unresectable hepatocellular carcinoma--a strategy to increase resectability. Ann Surg Oncol 2007;14:3301-9.

62. Frenette CT, Osorio RC, Stark J, et al. Conventional TACE and drug-eluting bead TACE as locoregional therapy before orthotopic liver transplantation: comparison of explant pathologic response. Transplantation 2014;98:781-7.

63. Salem R, Gordon AC, Mouli S, et al. Y90 radioembolization significantly prolongs time to progression compared with chemoembolization in patients with hepatocellular carcinoma. Gastroenterology 2016;151:1155-63.e2.

64. Edeline J, Crouzet L, Campillo-Gimenez B, et al. Selective internal radiation therapy compared with sorafenib for hepatocellular carcinoma with portal vein thrombosis. Eur J Nucl Med Mol Imaging. 2016;43:635-43.

65. Iñarrairaegui $M$, Thurston KG, Bilbao JI, et al. Radioembolization with use of yttrium-90 resin microspheres in patients with hepatocellular carcinoma and portal vein thrombosis. J Vasc Interv Radiol 2010;21:1205-12.

66. Gaba RC, Lewandowski RJ, Kulik LM, et al. Radiation lobectomy: preliminary findings of hepatic volumetric response to lobar yttrium-90 radioembolization. Ann Surg Oncol 2009;16:1587-96.

67. Vouche M, Lewandowski RJ, Atassi R, et al. Radiation lobectomy: time-dependent analysis of future liver remnant volume in unresectable liver cancer as a bridge to resection. J Hepatol 2013;59:1029-36.

68. Lewandowski RJ, Kulik LM, Riaz A, et al. A comparative analysis of transarterial downstaging for hepatocellular carcinoma: chemoembolization versus radioembolization. Am J Transplant 2009;9:1920-8.

69. Simonetti RG, Liberati A, Angiolini C, et al. Treatment of hepatocellular carcinoma: a systematic review of randomized controlled trials. Ann Oncol 1997;8:117-36.

70. Zaanan A, Williet N, Hebbar M, et al. Gemcitabine plus oxaliplatin in advanced hepatocellular carcinoma: a large multicenter AGEO study. J Hepatol 2013;58:81-8.

71. Leung TW, Patt YZ, Lau WY, et al. Complete

Cite this article as: Sahin IH, Khalil L, Millett R, Kaseb A. Neoadjuvant and adjuvant treatment approaches for hepatocellular carcinoma: future outlook. Chin Clin Oncol 2021;10(1):7. doi: 10.21037/cco-20-248 pathological remission is possible with systemic combination chemotherapy for inoperable hepatocellular carcinoma. Clin Cancer Res 1999;5:1676-81.

72. Yeo W, Mok TS, Zee B, et al. A randomized phase III study of doxorubicin versus cisplatin/interferon alpha2b/doxorubicin/fluorouracil (PIAF) combination chemotherapy for unresectable hepatocellular carcinoma. J Natl Cancer Inst 2005;97:1532-8.

73. Kaseb AO, Shindoh J, Patt YZ, et al. Modified cisplatin/ interferon $\alpha$-2b/doxorubicin/5-fluorouracil (PIAF) chemotherapy in patients with no hepatitis or cirrhosis is associated with improved response rate, resectability, and survival of initially unresectable hepatocellular carcinoma. Cancer 2013;119:3334-42.

74. Vitale A, Volk ML, Pastorelli D, et al. Use of sorafenib in patients with hepatocellular carcinoma before liver transplantation: a cost-benefit analysis while awaiting data on sorafenib safety. Hepatology 2010;51:165-73.

75. Vagefi PA, Hirose R. Downstaging of hepatocellular carcinoma prior to liver transplant: is there a role for adjuvant sorafenib in locoregional therapy? J Gastrointest Cancer 2010;41:217-20.

76. Golse N, Radenne S, Rode A, et al. Liver transplantation after neoadjuvant sorafenib therapy: preliminary experience and literature review. Exp Clin Transplant 2018;16:227-36.

77. Eilard MS, Andersson M, Naredi P, et al. A prospective clinical trial on sorafenib treatment of hepatocellular carcinoma before liver transplantation. BMC Cancer 2019;19:568.

78. Liu L, Chen H, Wang M, et al. Combination therapy of sorafenib and TACE for unresectable HCC: a systematic review and meta-analysis. PLoS One 2014;9:e91124.

79. Finn RS, Qin S, Ikeda M, et al. Atezolizumab plus Bevacizumab in Unresectable Hepatocellular Carcinoma. N Engl J Med 2020;382:1894-905.

80. Finn RS, Ryoo BY, Merle, P, et al. Results of KEYNOTE-240: phase 3 study of pembrolizumab (pembro) vs best supportive care (BSC) for second lien therapy in advanced hepatocellular carcinoma (HCC). J Clin Oncol 2019;37:4004. 